\section{Metabolomics for rheumatic diseases: has the time come?}

\author{
Luca Semerano, ${ }^{1,2,3}$ Paul-Henri Roméo, ${ }^{4}$ Marie-Christophe Boissier ${ }^{1,2,3}$
}

The last decade of biomedical research has been impacted by the appearance of systems biology, an analytical approach to complex biological systems that makes use of the '-omics', that is, high-throughput technologies that allow the comprehensive characterisation of genes (genomics), RNA transcripts (transcriptomics) or proteins (proteomics). Since their appearance, the 'omics' held promise of a radical change in the research paradigms. The classical research strategy adopts a 'bottom-up' approach, that is, a scientific hypothesis is tested in an isolated system (a cell type, a tissue) or in an animal model, and then extrapolated to the systemic level. Conversely, systems biology uses a 'top-down' approach in which data are gathered at systemic level and scientific hypotheses are subsequently derived and tested in a controlled, limited setting. ${ }^{1}$ The latter approach has been defined as datadriven or hypothesis-forming. Although genomics, transcriptomics and proteomics have generated a large amount of data, translation of these data into useful information, and in turn into beneficial outcomes for the patient, is only starting. ${ }^{2}$

The newcomer in the -omics family is metabolomics. The metabolome is defined as the complete set of metabolites present in a given biological system. Complementary to transcriptomics or proteomics, the metabolome analysis describes both qualitatively and quantitatively the final products of cellular regulatory pathways. As such, the metabolome reflects the ultimate response of a biological system to genetic and/or environmental changes.

Metabolites that are characterised in metabolomics studies are small molecules with size ranging from 100 to $1000 \mathrm{Da}^{3}$ Although the concept that metabolites in biological fluids may reflect health status

${ }^{1}$ Inserm UMR 1125, Bobigny, France; ${ }^{2}$ Sorbonne Paris Cité-Université Paris 13, Bobigny, France; ${ }^{3}$ Assistance Publique-Hôpitaux de Paris (AP-HP) Groupe hospitalier Avicenne-Jean Verdier-René Muret, Service de Rhumatologie, Bobigny, France; ${ }^{4}$ LRTS/DSV/ CEA, UMR967, Inserm, CEA, Paris-Diderot, Paris-Sud, Fontenay-aux-Roses, Cedex, France

Correspondence to Professor Marie-Christophe Boissier, Inserm UMR 1125, 74 rue Marcel Cachin, Bobigny 93000, France; marie-chistophe.boissier@avc. aphp.fr dates back to the 1940 s, the term 'metabolic profile' was only introduced in the early 1970s when metabolite characterisation entered into the era of gas chromatography coupled to mass spectrometry technology. ${ }^{4}$ Extensive metabolomic analyses have become possible only recently thanks to the use of mass spectrometry or nuclear MR coupled to multivariate analyses of the generated data. The actual version of the human metabolome database 3.6 refers to 41808 metabolic entries, ${ }^{5} 6$ and ongoing developments in microfluidic and intergrated interfaces will boost the human metabolome project in the next few years. ${ }^{7}$

Metabolomics might be well suited to study rheumatic conditions for several reasons. First, metabolites can be considered as 'final' responders to environmental cues and thus metabolomics will indicate biological responses of patients suffering from rheumatic conditions to environment, including nutrition, disease states, infection, exposition to xenobiotics, pharmacological treatments and others. ${ }^{8}$ Second, pathways that regulate metabolic homeostasis and immune response display an interplay that is evolutionary conserved. ${ }^{9}$ Metabolic diseases like obesity and type II diabetes are associated with chronic inflammation, and the high burden of cardiovascular disease in rheumatic conditions shows that immunoinflammatory diseases are associated with altered metabolic homeostasis. Indeed, proliferating cells involved in inflammation and immune response act in an environment of low-oxygen tension and rely on anaerobic glucose metabolism for their nutritional supply. Moreover, their proliferative and effector activity affects the metabolism of the involved tissues. This can reflect into modifications of the metabolic profile of the patient that will be depicted by metabolomics. ${ }^{10}$

But, rather than depicting metabolic repercussion of inflammation, the real challenge of metabolomics in rheumatology will be to identify metabolite profiles that are specific to a given rheumatic condition. For example, in a Swedish study, plasma metabolomics discriminated patients with rheumatoid arthritis (RA) or psoriatic arthritis (PsA) from controls with $90 \%$ sensitivity and $94 \%$ specificity. ${ }^{11}$
In another work, the urinary metabolic footprint of patients with RA or PsA predicted clinical response to anti-tumour necrosis factor agents with $88.9 \%$ sensitivity and $85.7 \%$ specificity. Moreover, response to treatment with either infliximab or etanercept reflected into a different metabolite profile. ${ }^{12}$

These results indicate that metabolomics may provide relevant biomarkers to improve diagnostic accuracy, define prognosis and predict and monitor treatment efficacy. ${ }^{13}$ Moreover, disease-specific metabolic pathways could indicate new potential therapeutic targets as acting on these pathways may allow to modulate the disease process by directly altering the metabolism of the involved cellular actors. Additionally, some metabolites may be endowed themselves with a pathological action, as is the case of arachidonic acid metabolites that are produced in response to proinflammatory cues and that further potentiate and amplify the inflammatory process. For example, synovial cells from patients with RA have been reported to preferentially convert oestrogens to $16 \mathrm{OH} \alpha$-metabolites, which may facilitate synovial inflammation, ${ }^{14}$ while the cholesterol metabolite 27-hydroxycholesterol promotes vessel wall inflammation and atherosclerosis. ${ }^{15}$

Finally, metabolomics comes at a time when the other omics techniques are well developed and advanced, which would allow to implement and integrate the data obtained from metabolic fingerprinting with those coming from the other three omics, forming in the -omics world the group of the 'big four'. 2 This has been claimed as the inevitable way to validate metabolomics results, together with the replication of the results in independent cohorts.

Nevertheless, the systems biology approach does not explain the reason for the abundance of a given metabolite in a biological system, nor establish whether the metabolite level is associated with the cause or the consequence of the disease. ${ }^{16}$ For example, in a model of experimental colitis, serum glutamine prevalence over other amino acids correlated with the degree of colonic inflammation. Of note, glutamine supplementation ameliorated colonic lesions. ${ }^{17}$ This demonstrated that glutamine increased as a result of a compensatory and not of a pathogenic mechanism, and that, for therapeutic purposes, this increase needs to be enhanced and not antagonised.

Hence, we strongly support the idea that classical 'bottom-up' research can be usefully integrated with systems biology to dissect a metabolic pathway responsible 
for the increased or reduced presence of a given metabolite in a sample. Guma et $a l^{18}$ used a similar approach. To study the therapeutic potential of choline kinase- $\alpha(\mathrm{ChoK} \alpha)$ pathways inhibition in $\mathrm{RA}$, the authors performed targeted ${ }^{1} \mathrm{H}$ magnetic resonance spectroscopy (HMRS) on both synovium samples and cultured fibroblast-like synoviocytes (FLS) from patients with RA and showed the presence of high levels of choline-containing metabolites. Moreover, the ratio of glycerophosphocholine to phosphocholine in the samples suggested that these metabolites resulted from ChoK $\alpha$ activity. Then, they confirmed the hypothesis of ChoK $\alpha$ activation by repeating HMRS profiling of FLS in the presence or absence of ChoK $\alpha$ inhibitors. Finally, the authors confirmed the therapeutic relevance of ChoK $\alpha$ pathways by showing that the ChoK $\alpha$ inhibitor MN58b ameliorated arthritis and resulted in reduced synovial transcription of interleukin (IL)-1, IL-6 and matrix metalloproteinases in the $\mathrm{K} / \mathrm{BxN}$ mouse model. In this work, the metabolites of interest had been chosen previously, hence a proper metabolomics analysis was not performed. Nevertheless, the rationale for targeting ChoK pathways in RA was derived from metabolomics studies performed in cancer. ${ }^{19}$ For this reason, the work of Guma et al allows to foresee how, in the near future, larger-scale studies may integrate modern high-throughput technologies with classical hypothesis testing in order to dissect the role of metabolism in chronic inflammatory disease.

In fact, we forecast that future advances of metabolomics in biomedical research will be particularly useful in the domain of rheumatic diseases, both for the identifications of biomarkers and for new therapeutic targets. The application of metabolomics (and other -omics) to large-scale epidemiological research, with longitudinal observation and interfaces between databases, will allow to determine the relevance of metabolite footprints for disease, even at the individual level. The availability of shared, low-cost analytical platforms and the collaborations between research teams with different expertise and competences are critical points for further progress in this emerging field. ${ }^{20}$

Contributors $L S$ and $M-C B$, drafted the article. $L S, P-H R$ and $M-C B$ revised the article for important intellectual content. LS, P-HR and M-CB approved the final version.

\section{Competing interests None.}

Provenance and peer review Commissioned; externally peer reviewed.

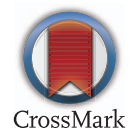

To cite Semerano L, Roméo P-H, Boissier M-C. Ann Rheum Dis 2015;74:1325-1326.

Received 5 November 2014

Revised 22 December 2014

Accepted 6 January 2015

Published Online First 21 January 2015

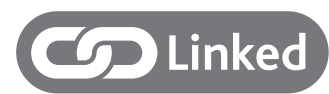

- http://dx.doi.org/10.1136/annrheumdis-2014205696

Ann Rheum Dis 2015;74:1325-1326.

doi:10.1136/annrheumdis-2014-206618

\section{REFERENCES}

1 Nicholson JK, Lindon JC. Systems biology: metabonomics. Nature 2008;455:1054-6.

2 Veenstra TD. Metabolomics: the final frontier? Genome Med 2012;4:40.

3 Holmes E, Wilson ID, Nicholson JK. Metabolic phenotyping in health and disease. Cell 2008;134:714-17.

4 Horning EC, Horning MG. Metabolic profiles: gas-phase methods for analysis of metabolites. Clin Chem 1971;17:802-9.
5 http://www.hmdb.cal, last consultation October 29th 2014

6 Wishart DS, Jewison T, Guo AC, et al. HMDB 3.0the human metabolome database in 2013. Nucleic Acids Res 2013;41:D801-7.

7 Fuhrer T, Zamboni N. High-throughput discovery metabolomics. Curr Opin Biotechnol 2014;31C:73-8.

8 Clayton TA, Lindon JC, Cloarec 0 , et al. Pharmacometabonomic phenotyping and personalized drug treatment. Nature 2006;440:1073-7.

9 Hotamisligil GS. Inflammation and metabolic disorders. Nature 2006;444:860-7.

10 Fitzpatrick M, Young SP. Metabolomics-a nove window into inflammatory disease. Swiss Med Wkly 2013:143:w13743.

11 Madsen RK, Lundstedt T, Gabrielsson J, et al. Diagnostic properties of metabolic perturbations in rheumatoid arthritis. Arthritis Res Ther 2011; 13:R19.

12 Kapoor SR, Filer A, Fitzpatrick MA, et al. Metabolic profiling predicts response to anti-tumor necrosis factor $\alpha$ therapy in patients with rheumatoid arthritis. Arthritis Rheum 2013:65:1448-56.

13 Tektonidou MG, Ward MM. Validity of clinical associations of biomarkers in translational research studies: the case of systemic autoimmune diseases. Arthritis Res Ther 2010;12:R179.

14 Schmidt M, Hartung R, Capellino $\mathrm{S}$, et al. Estrone/ 17beta-estradiol conversion to, and tumor necrosis factor inhibition by, estrogen metabolites in synovial cells of patients with rheumatoid arthritis and patients with osteoarthritis. Arthritis Rheum 2009;60: 2913-22.

15 Umetani M, Ghosh P, Ishikawa T, et al. The cholesterol metabolite 27-hydroxycholesterol promotes atherosclerosis via proinflammatory processes mediated by estrogen receptor alpha. Cell Metab 2014;20:172-82.

16 Arakaki AK, Skolnick J, McDonald JF. Marker metabolites can be therapeutic targets as well. Nature 2008;456:443.

17 Shiomi Y, Nishiumi S, Ooi M, et al. GCMS-based metabolomic study in mice with colitis induced by dextran sulfate sodium. Inflamm Bowel Dis 2011;17:2261-74

18 Guma M, Sanchez-Lopez E, Lodi A, et al. Choline kinase inhibition in rheumatoid arthritis. Ann Rheum Dis 2015:74:1399-1407.

19 Glunde K, Serkova NJ. Therapeutic targets and biomarkers identified in cancer choline phospholipid metabolism. Pharmacogenomics 2006;7:1109-23.

20 Tzoulaki I, Ebbels TM, Valdes A, et al. Design and analysis of metabolomics studies in epidemiologic research: a primer on -omic technologies. Am J Epidemiol 2014;180:129-39. 